\title{
High-resolution observations of SDSS J080800.99+483807.7 in the optical and radio domains
}

\section{A possible example of jet-triggered star formation}

\author{
Y. E. Rashed ${ }^{1,2}$, J. Zuther ${ }^{1}$, A. Eckart ${ }^{1,3}$, G. Busch ${ }^{1}$, M. Valencia-S. ${ }^{1}$, M. Vitale ${ }^{1,3}$, S. Britzen ${ }^{3}$, and T. Muxlow ${ }^{4}$ \\ 1 I. Physikalisches Institut, Universität zu Köln, Zülpicher Straße 77, 50937 Köln, Germany \\ 2 Department of Astronomy, Faculty of Science, University of Baghdad, 10071 Baghdad, Aljadirya, Iraq \\ e-mail: yasir@ph1.uni-koeln.de \\ 3 Max-Planck-Institut für Radioastronomie, Auf dem Hügel 69, 53121 Bonn, Germany \\ ${ }^{4}$ MERLIN/VLBI National Facility, Jodrell Bank Observatory, University of Manchester, Macclesfield, Cheshire SK11 9DL, UK
}

Received 4 July 2013 / Accepted 24 July 2013

\section{ABSTRACT}

\begin{abstract}
Context. Double-lobe radio galaxies are ideally suited to investigate the interaction of the individual components of the radio structure with the intergalactic medium and the interstellar medium of the host galaxy. SDSS J080800.99+483807.7 has been serendipitously discovered in MERLIN $18 \mathrm{~cm}$ observations to be a double-lobed radio galaxy. Because it is an optically faint source, basic information like redshift, linear size, and structure has been incomplete until now. Furthermore, there are no spectra of this source available in any databases.

Aims. The goal of this work is to derive the main physical properties of SDSS J080800.99+483807.7 and study the possible interaction between the radio jets and the interstellar medium of the host galaxy.

Methods. To achieve this goal, we used optical spectroscopy and radio interferometry. The radio data were obtained with MERLIN at $18 \mathrm{~cm}$ and the optical data with the Multi-Object Double Spectrograph (MODS) at the Large Binocular Telescope (LBT).

Results. The redshift of the galaxy is $z=0.2805 \pm 0.0003$, resulting in a linear size of the observed radio structure of $\sim 26.3 \mathrm{kpc}$. The optical line emission as well as the infrared and radio continuum emission suggest a high star-formation activity. In addition, we estimated the mass of the central black hole to be $\log \left(M_{\mathrm{BH}} / M_{\odot}\right) \approx 6.9$.

Conclusions. The MODS spectrum and the optical images from Sloan Digital Sky Survey suggest that SDSS J080800.99+483807.7 is an elliptical host galaxy. In combination with the overall radio structure, we argue that the star formation could be the result of the back-flow along the jet and the interstellar medium of the host.
\end{abstract}

Key words. galaxies: nuclei - radio continuum: galaxies - techniques: spectroscopic

\section{Introduction}

The first observations of radio galaxies have provided very strong evidence that there are extended jets flowing from the center of some of these sources, spanning a few kilo parsec to a few mega parsecs (e.g., Bridle \& Perley 1984, and references therein). The jets appear to be associated with the central massive black hole and the accretion disk (e.g., Begelman et al. 1984; Blandford \& Znajek 1977; Blandford \& Payne 1982). It has been widely speculated about the mechanisms for fueling the central massive black hole with matter from the host galaxy and the impact of nuclear activity on the host (i.e., radiation field, outflows, jets, etc.). Fabian (2012) reports on observational evidence for feedback between the radio jet and the interstellar/intergalactic medium, especially in the radio/kinematic mode in which a significant back-flow of material along the periphery of the jet or outflow onto the host occurs (Wagner et al. 2013; Silk 2013). Wagner et al. (2013) use three-dimensional gridbased hydrodynamical simulations to show that ultrafast outflows from active galactic nuclei (AGN) result in considerable feedback of energy and momentum into the interstellar medium (ISM) of the host galaxy. Silk (2013) shows that the AGNinduced pressure, which is caused by jets and/or winds that flow back onto a gas-rich host, can lead to pressure-regulated star formation with significantly enhanced star-formation rates. The back-flow phenomenon has also been discussed, especially in the case of X-shaped radio galaxies (XRGs), which are a special type of radio galaxies with two pairs of lobes (one active, bright pair and a second fainter, more diffuse pair; Leahy \& Williams 1984). For a radio galaxy to be classified as an XRG, it needs to have the two pairs of jets strongly misaligned and flowing from the center of the source; the minor pair must flow in another direction as the major pair, otherwise it would be double-double radio galaxy (DDRG; e.g. Brocksopp et al. 2011, and references therein). One finds that 5\%-10\% of Fanaroff-Riley type II radio galaxies are X-shaped galaxies (Fanaroff \& Riley 1974). There are several competing interpretations of the physical nature of the radio morphology, e.g., black-hole spin reorientation, plasma back-flows from the lobes, binary black holes, and jet ISM interaction (cf. Gopal-Krishna et al. 2012, for a review). The nuclei of XRGs, which presumably represent the transition population between FR I and FR II radio sources (e.g., Landt et al. 2010), are valuable probes of the interaction between the black hole, its jet, and the host (e.g., Hodges-Kluck \& Reynolds 2011; Wagner \& Bicknell 2011).

In our MERLIN $18 \mathrm{~cm}$ data set of $4 \mathrm{C} 48.21$ we have serendipitously identified SDSS J080800.99+483807.7 (in the following J0808) as a rather compact double-lobed radio galaxy. 
Such objects are particularly interesting since they could be small due to extreme foreshortening or an actual small size. In case of an extreme foreshortening, one would expect the radio jet to be pointed towards the observer under a small angle with respect to the line of sight, such that the central nucleus appears to be bright due to relativistic beaming. In the latter case, one of the lobes is also expected to be brighter and possibly cover the central source due to its extent. However, J0808 appears as a symmetric double-lobed source with a nuclear component that is very weak with respect to the lobes and somewhat more extended than compact. Hence, we can assume that its small overall apparent size is due to a small physical size. Especially for physically small sources, a back-flow towards the center along the jets is expected, resulting in an interaction at the nucleus. The possibility of back-flow interactions has also been reported (cf. Hodges-Kluck \& Reynolds 2011) for X-shaped radio sources. In particular, we complement the radio information with optical spectroscopy of the nuclear region of the galaxy and investigate the possibility of triggered star formation. A detailed description of the observations and the J0808 data reduction in this context is given in Sect. 2. In Sect. 3 we discuss the results: the optical spectrum of the source (Sect. 3.1), the radio structure and its linear size (Sect. 3.2), the radio loudness of J0808 (Sect. 3.3), the central black hole (Sect. 3.4), and finally in Sect. 3.5, the star formation in the host of J0808. A discussion follows in Sect. 4.

\section{Observation and data reduction}

In this section, we describe the radio and spectroscopic observations, the data reduction, and the complementary data from public archives used in our analysis.

\subsection{MERLIN $18 \mathrm{~cm}$ observation}

The Multi-Element Radio Linked Interferometer Network (MERLIN) is an interferometer array of radio telescopes distributed across England with baselines up to $217 \mathrm{~km}$ length. The $18 \mathrm{~cm}$ observations were carried out in May 2005. The resulting angular resolution is about $0.25 \times 0 . ' 15$ with a position angle of $1.5^{\circ}$. The total integration time on J0808 was about $9.2 \mathrm{~h}$ and the noise in the image is about $0.1 \mathrm{mJy}^{\text {beam }}{ }^{-1}$. The central radio component is located within 0 .' 1 of the position of the optical source J0808. Figure 1 presents the image of the radio source. Details of the radio observation and data reduction are given in Zuther et al. (2012). An extended structure at the central source component of J0808 gives some evidence for a possible backflow towards the center that may result in an interaction with the ISM at the nucleus. In fact, J0808 shows extended emission along a position angle that is by at least $50^{\circ}$ different from that of the prominent double-lobe structure. Signatures of the peculiar shape are prominent on contour lines as high as at least five times the noise level in the original and the smoothed version of the radio image shown in Fig. 1. This is a signature well known for XRGs or in general objects that are subject to a significant back-flow along the radio jet onto the host galaxy (Wagner et al. 2013; Silk 2013).

\subsection{Multi-Object Double Spectrograph observation}

The Multi-Object Double Spectrograph (MODS) is mounted at the Large Binocular Telescope (LBT), located on Mount Graham in the Pinaleno Mountains, USA. It operates between 3200 and
$10500 \AA$ at a nominal spectral resolution of $\lambda / \delta \lambda \approx 2000$ (i.e., $\sim 150 \mathrm{~km} \mathrm{~s}^{-1}$ ). Its field of view is about $6 \times 6 \mathrm{arcmin}^{2}$. Furthermore, imaging, long-slit, and multi-object spectroscopy can be used in this instrument, with a slit aperture of 0.6 . The MODS1 spectrograph had first light in September 2010 and began its science operation in September 2011. The MODS chargecoupled device (CCD) detector is an e2vCCD231-68 $8 k \times 3 k$ with a $15 \mu \mathrm{m}$ pixel pitch. For the grating spectroscopy, the full spectral range was split into a blue and red channel, which are 3200-6000 $\AA$ (blue) and 5000-10500 $\AA$ (red) (Pogge et al. 2010,2012 ). For a slit width of $0 .{ }^{\prime} 6$ and a redshift of $z \approx 0.28$, we probe the central $2.5 \mathrm{kpc}$ region of the host.

For data reduction, we used the modsCCDRed software provided for MODS1, built in Python. This software package allows us to perform bias correction, flat fielding, and other standard steps of data reduction.

As a first step, we prepared normalized spectral flat field images for each channel (blue and red) through the following ${ }^{1}$ :

- corrected the bias and trimmed the flat fields images;

- median combination of the bias-corrected flats;

- repaired the bad columns using the bad pixel lists for the detector;

- eliminated the color term to produce a normalized "pixel flat".

In a second step, we applied these two normalized spectral flat fielding images to all raw science and calibration frames. In a third step, we performed the wavelength calibration for science frames using the lamp frames provided by the instrument for different elements (argon, neon, xenon and krypton). Here, we used the IRAF reduction package to identify the lamp lines and then transformed the science frames accordingly. In addition, we tested the calibration using the $\mathrm{OH}$-skyline atlas (Osterbrock et al. 1996, 1997). We found both ways of calibration to be in good agreement. Then, in a final step we extracted one-dimensional spectra from the two-dimensional images and flux-calibrated them using the standard star G191B2B.

\subsection{The Sloan Digital Sky Survey}

The Sloan Digital Sky Survey (SDSS) is a sensitive optical imaging and spectroscopic public survey of about $10^{4}$ square degrees of the northern sky (York et al. 2000). It provides images and photometric parameters in five bands $(u, g, r, i$, and $z)$ at an average seeing of 1."5 and down to a limiting magnitude of $\sim 22.2$ in $r$ band. The $R \sim 2000$ spectroscopy covers a wavelength range from 3800-9200 ^. SDSS began its operation in May 2000. We used the seventh data release of the SDSS (Abazajian et al. 2009). Magnitudes for J0808 in the five SDSS bands are given in Table 1. In Fig. 2, we show the $i$-band SDSS optical image of the galaxy.

\subsection{The Wide-field Infrared Survey Explorer}

The Wide-field Infrared Survey Explorer (WISE) was a NASA infrared-wavelength astronomical space telescope, which was operated between December 2009 to February 2011 (Wright et al. 2010). It performed an all-sky astronomical survey with images in wavelength bands at $3.4 \mu \mathrm{m}, 4.6 \mu \mathrm{m}, 12 \mu \mathrm{m}$, and $22 \mu \mathrm{m}$, using a $40 \mathrm{~cm}$ diameter infrared telescope in Earth orbit. In the

1 The user manual can be found at http://www.astronomy. ohio-state.edu/MODS/Manuals/MODSCCDRed.pdf 


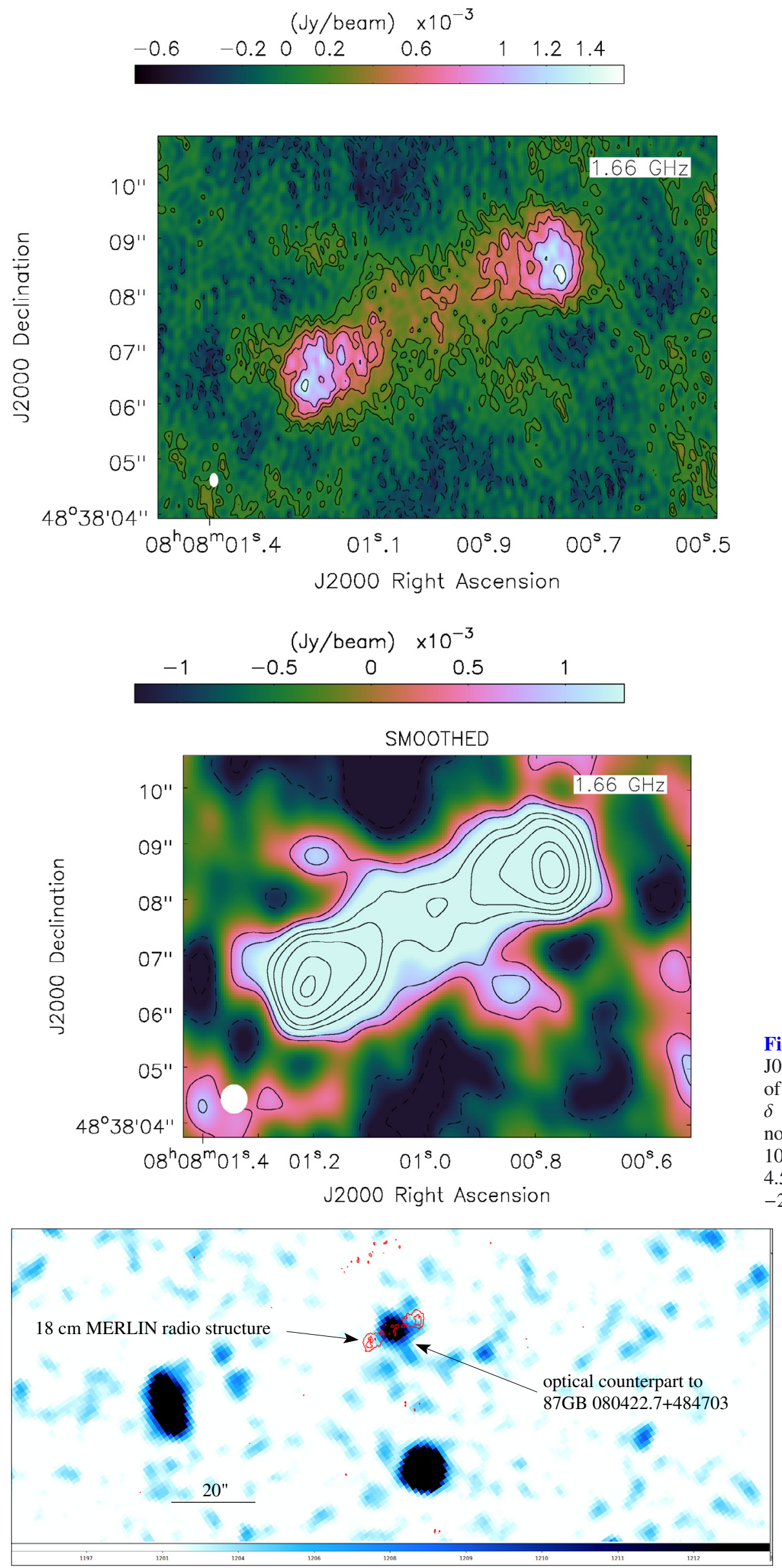

Fig. 1. $18 \mathrm{~cm}$ MERLIN image of SDSS J080800.99+483807.7. The J2000 coordinates of the central source are $\alpha=08: 08: 00.99$ and $\delta=+48: 38: 07.73$. Top: original image at a noise level of about 1.1e-4 Jy/beam. Bottom: 10 pix smoothed image at a noise level of about $4.5 \mathrm{e}-4 \mathrm{Jy} / \mathrm{beam}$. The contour levels are each at -3 , $-2,1,2,4,5.5,8,12,14$ times the noise level.

Fig. 2. SDSS image of J0808, overlaid with MERLIN $18 \mathrm{~cm}$ contours in red. 


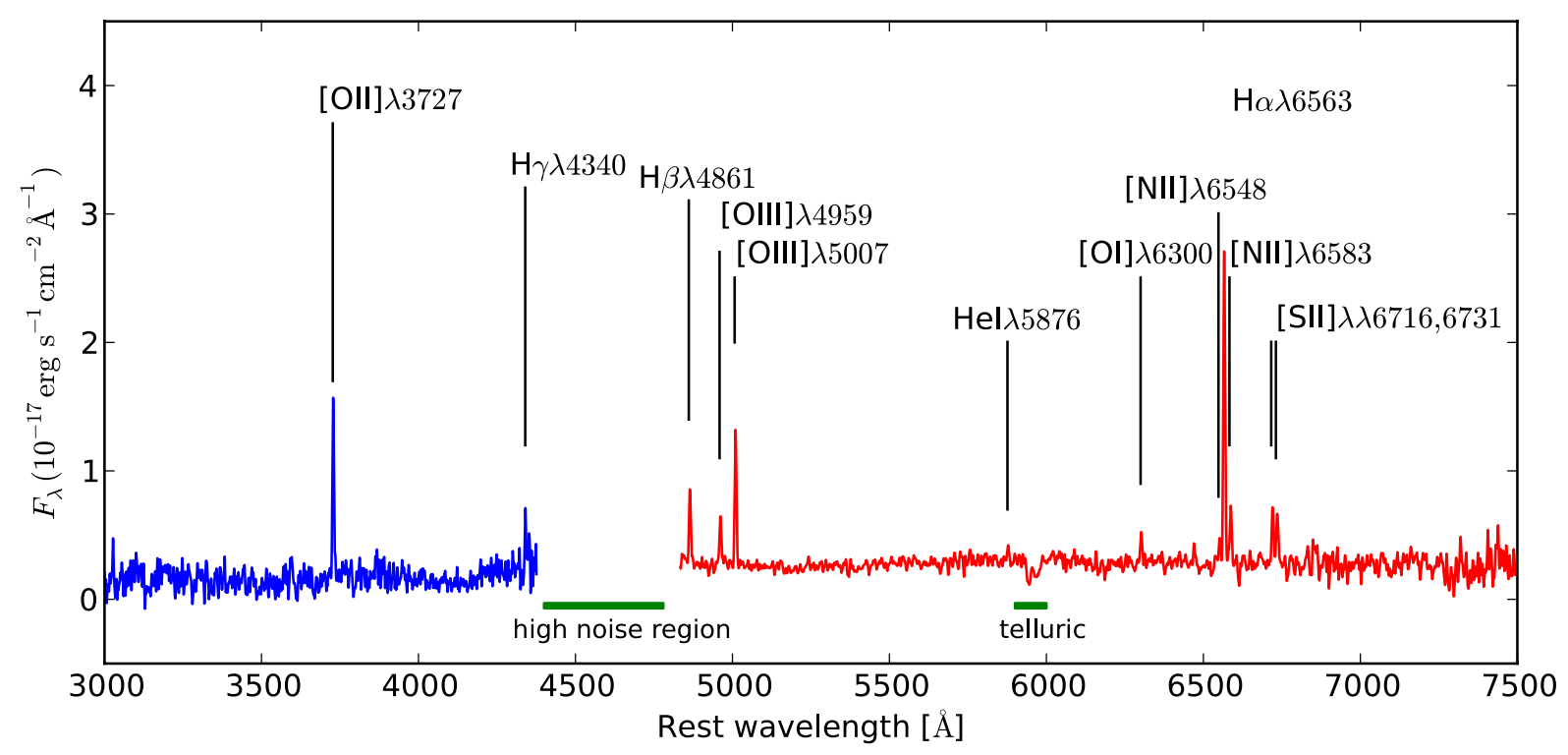

Fig. 3. Optical spectrum of J0808. The blue and red spectra represent the data from the respective channel. A gap between the two channels that has been excluded because of high noise and a region that shows telluric features are marked.

Table 1. Photometric measurements of J0808 in the SDSS and WISE photometric systems.

\begin{tabular}{ccc}
\hline \hline Survey & Band & Magnitude \\
\hline SDSS & $u$ & $23.68 \pm 1.35$ \\
SDSS & $g$ & $24.05 \pm 0.91$ \\
SDSS & $r$ & $21.9 \pm 0.20$ \\
SDSS & $i$ & $20.52 \pm 0.10$ \\
SDSS & $z$ & $19.70 \pm 0.15$ \\
WISE & $3.4 \mu \mathrm{m}$ & $15.61 \pm 0.06$ \\
WISE & $4.6 \mu \mathrm{m}$ & $15.51 \pm 0.15$ \\
WISE & $12 \mu \mathrm{m}$ & $12.30 \pm 2.50$ \\
\hline
\end{tabular}

all-sky data release from March 14, 2012, the source J0808 is detected at all bands, except at $22 \mu \mathrm{m}$. The flux densities are listed in Table 1 . The uncertainty of the $12 \mu \mathrm{m}$ flux density was estimated from the corresponding image provided by the data release.

\section{Results}

\subsection{Measurement of emission-lines}

We present the reduced optical spectrum in Fig. 3. Prominent emission lines have been fitted with the spectral analysis tool Specview assuming Gaussian shape. Positions, resulting redshifts, flux density, and full-width at half maximum (FWHM) of the emission lines are listed in Table 2.

The emission-line diagnostic diagram delivers information about the dominant ionization mechanism of the gas in the studied region. Studies of different samples of narrow emission-line galaxies have also shown some trends in the diagrams that are associated with stellar mass, metallicity and possibly morphology of the host. No reddening corrections has to be applied to calculate line ratios in these diagrams since the emission lines are close to each other in wavelength:

- $[\mathrm{N} \mathrm{II}] / \mathrm{H} \alpha$ vs. $[\mathrm{O}$ III] $] / \mathrm{H} \beta$ (Veilleux \& Osterbrock 1987; Kauffmann et al. 2003; Kewley et al. 2001, 2003; Lamareille et al. 2004; Lamareille 2010; Juneau et al. 2011).
- $[\mathrm{S}$ II $] / \mathrm{H} \alpha$ vs. $[\mathrm{O}$ III $] / \mathrm{H} \beta$ (Veilleux \& Osterbrock 1987; Kewley et al. 2001, 2006; Lamareille et al. 2004; Lamareille 2010; Juneau et al. 2011).

- $[\mathrm{O} \mathrm{I}] / \mathrm{H} \alpha$ vs. [O III] $/ \mathrm{H} \beta$ (Kewley et al. 2001, 2006).

The measured ratios between emission lines are:

- $\log \frac{[\mathrm{O} \mathrm{III}] \lambda 5007}{\mathrm{H} \beta}=0.337 \pm 0.006$;

- $\log \frac{[\mathrm{NII}] \lambda 6583}{\mathrm{H}_{\alpha}}=-0.822 \pm 0.005$;

- $\log \frac{[\mathrm{S} \mathrm{II}] \lambda 6716, \lambda 6731}{\mathrm{H} \alpha}=-0.448 \pm 0.006$;

$-\log \frac{[\mathrm{OI}] \lambda 6300}{\mathrm{H} \alpha}=-1.090 \pm 0.010$.

For our target source only an estimate of a photometric redshift of $z \sim 0.7$ was available from the SDSS. To derive the spectroscopic redshift, we first reduced the data as outlined in the previous section. The optical spectrum is shown in Fig. 3. For the emission lines evident in Fig. 3 and listed in Table 2, we used the equation

$z=\frac{\lambda_{\mathrm{obs}}}{\lambda_{\text {rest }}}-1$

and calculated the redshift for each line as shown in Table 2. The mean redshift and its standard deviation are $z=0.2805 \pm$ 0.0003. Combining the redshift with the cosmology constants $H_{0}=70 \mathrm{~km} \mathrm{~s}^{-1} \mathrm{Mpc}^{-1}, \Omega_{\mathrm{m}}=0.3$, and $\Omega_{\Lambda}=0.7$ (Spergel et al. 2003, which we use throughout the paper), we calculated the luminosity distance $D_{\mathrm{L}}$ (Hogg 1999) to be $1437.2 \mathrm{Mpc}$. This corresponds to a linear-size scale conversion factor of $6.98 \mathrm{kpc}$ per arcsecond.

As we see in the diagnostic diagrams (Fig. 4), J0808 is located in the region characteristic for host galaxies with high star-formation rates ([N II]-based diagram) and a tendency to have contributions to line emission from an active nucleus ([O I]-based diagram). J0808 is located closer to the region of star formation and low excitation galaxies, as compared to high excitation galaxies by Kunert-Bajraszewska \& Thomasson (2009). In particular, the low $[\mathrm{N} \mathrm{II}] / \mathrm{H} \alpha$ also suggests a low metallicity. The diagnostic diagrams in Fig. 12 by Vitale et al. (2012) suggest a thermal rather than a nonthermal origin of the optical emission lines. From the optical images it is difficult 
Table 2. Observed emission lines: wavelengths, redshifts, flux densities, and line widths.

\begin{tabular}{|c|c|c|c|c|c|}
\hline Channel & Line & $\begin{array}{c}\text { Wavelength } \\
{[\AA]}\end{array}$ & Redshift & $\begin{array}{c}\text { Flux } \\
{\left[10^{-17} \mathrm{erg} \mathrm{s}^{-1} \mathrm{~cm}^{-2}\right]}\end{array}$ & $\begin{array}{c}F W H M \\
{\left[\mathrm{~km} \mathrm{~s}^{-1}\right]}\end{array}$ \\
\hline Blue & [O II] $\lambda 3727$ & 4772.1 & 0.2804 & $18.80 \pm 0.07$ & $445 \pm 8$ \\
\hline Blue & $\mathrm{H} \gamma \lambda 4340$ & 5555.9 & 0.2801 & $4.01 \pm 0.13$ & $225 \pm 7$ \\
\hline Red & $\mathrm{H} \beta \lambda 4861$ & 6226.5 & 0.2809 & $6.91 \pm 0.04$ & $333 \pm 10$ \\
\hline Red & [O III] $\lambda 4959$ & 6350.9 & 0.2806 & $4.04 \pm 0.02$ & $345 \pm 10$ \\
\hline Red & [O III $] \lambda 5007$ & 6412.5 & 0.2807 & $15.03 \pm 0.02$ & $355 \pm 10$ \\
\hline Red & {$[\mathrm{He} \mathrm{I}] \lambda 5876$} & 7523.7 & 0.2804 & $1.51 \pm 0.04$ & $252 \pm 9$ \\
\hline Red & [O I] $\lambda 6300$ & 8066.4 & 0.2803 & $2.92 \pm 0.03$ & $277 \pm 8$ \\
\hline Red & {$[\mathrm{N}$ II $] \lambda 6548$} & 8386.1 & 0.2807 & $2.65 \pm 0.03$ & $302 \pm 8$ \\
\hline Red & $\mathrm{H} \alpha \lambda 6563$ & 8405.0 & 0.2806 & $36.02 \pm 0.03$ & $290 \pm 8$ \\
\hline Red & {$[\mathrm{N}$ II $] \lambda 6583$} & 8431.3 & 0.2807 & $5.42 \pm 0.03$ & $261 \pm 8$ \\
\hline Red & [S II] $\lambda 6716$ & 8602.1 & 0.2808 & $6.56 \pm 0.04$ & $290 \pm 8$ \\
\hline Red & [S II] $\lambda 6731$ & 8621.2 & 0.2808 & $6.25 \pm 0.04$ & $324 \pm 8$ \\
\hline
\end{tabular}
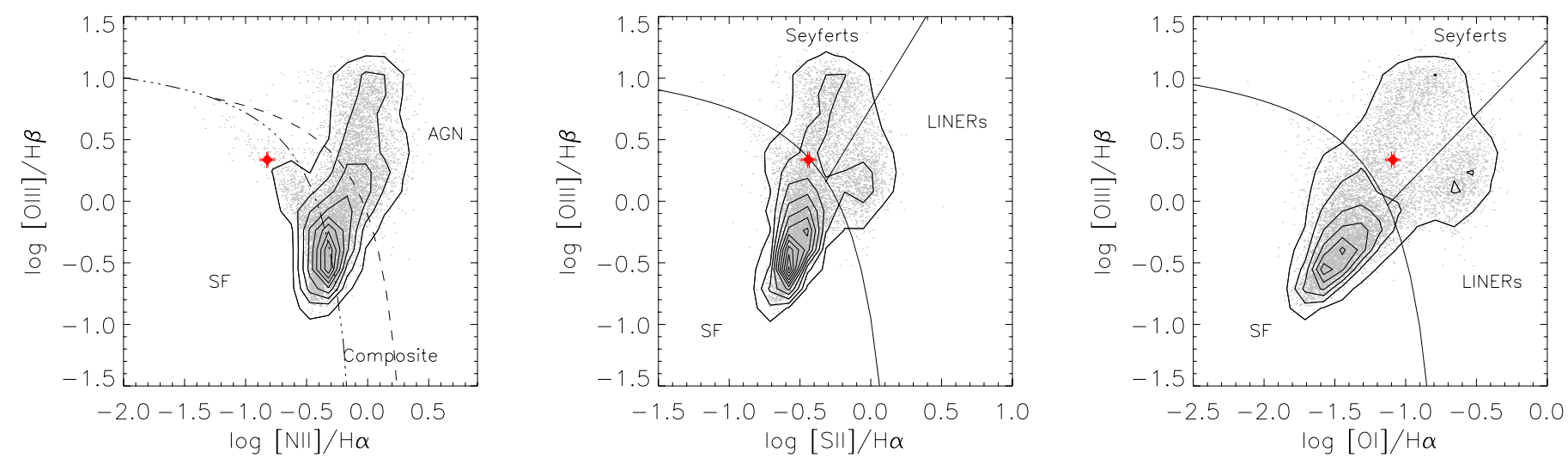

Fig. 4. Diagnostic diagrams $[\mathrm{N} \mathrm{II}] / \mathrm{H} \alpha$ vs. $[\mathrm{O} \mathrm{III}] / \mathrm{H} \beta$, $[\mathrm{S} \mathrm{II}] / \mathrm{H} \alpha$ vs. $[\mathrm{O} \mathrm{III}] / \mathrm{H} \beta$ and $[\mathrm{OI}] / \mathrm{H} \alpha$ vs. $[\mathrm{O} \mathrm{III}] / \mathrm{H} \beta$ for the radio emitters taken from Vitale et al. (2012). The contours show galaxy densities in steps of 80 galaxies per each additional contour. The position of the source analyzed here is shown as a red dot.

to provide a host galaxy classification. The host visible in the SDSS images is in agreement either with an elliptical or a bulge of a low-luminosity disk system. The low metallicity then suggests either an early evolutionary stage or an overall low mass of the host galaxy (Kauffmann et al. 2003; Vitale et al. 2013). Interpolating the $z$-band and $3.4 \mu \mathrm{m}$ flux densities, we get a $K$-band magnitude of about $K \sim 16.9$ and a flux density of $S_{K}=1.09 \times 10^{-4} \mathrm{Jy}$. We can estimate the old stellar mass via

$$
M_{*, \text { old }}\left[M_{\odot}\right]=2.6 \times 10^{8} D^{2}[\mathrm{Mpc}] S_{K}[\mathrm{Jy}]
$$

(Thronson \& Greenhouse 1988). This is equivalent to a population $K$-band $\mathrm{M} / \mathrm{L}$ ratio of about 23 and applying the $K$-band luminosity to flux density relation by Krabbe et al. (1994). Using a distance of $1437.2 \mathrm{Mpc}$, we then find a stellar mass of about $6 \times 10^{10} M_{\odot}$. This is one to two orders of magnitudes less than the mass of the Milky Way but about an order of magnitude more than the upper limit of $2 \times 10^{9} M_{\odot}$ derived for the stellar mass of the starburst galaxy M82 (Greco et al. 2012). M82 is known to have a strong, nuclear starburst driven wind over which a major portion of the newly formed metals can be lost. Hence, J0808 could be a low-mass low-metallicity elliptical.

\subsection{Linear size of the radio source}

Early investigations of the relation between the median linear size of the galaxy $D_{\text {med }}$ as a function of redshift $z$ (Eales 1985) resulted in $D_{\text {med }} \propto(1+z)^{-1.1 \pm 0.5}$ for $\Omega_{0}=0$, and $D_{\text {med }} \propto(1+$ $z)^{-1.45 \pm 0.4}$ for $\Omega_{0}=1$. Initially, there was no indication for a correlation between luminosity and linear size. However, Oort et al. (1987) present a strong correlation between $D_{\text {med }}$ and $z$ of $D_{\text {med }} \propto(1+z)^{-3.3 \pm 0.5}$ at $\Omega_{0}=0$. Follow-up investigations by Neeser et al. (1995) could not confirm such a strong correlation and resulted in an updated relation between the median size and redshift of $D_{\text {med }} \propto(1+z)^{-1.2 \pm 0.5}$ when $\Omega_{0}=0$ and $D_{\text {med }} \propto(1+$ $z)^{-1.7 \pm 0.4}$ when $\Omega_{0}=1$. In recent years, spectroscopic techniques have helped to understand the physical properties of more distant objects that appear to be largely unresolved. Using spectroscopic redshifts allows us to derive the linear size of the radio source host galaxies.

We derive a linear source size via

$l=\vartheta \times D_{\mathrm{A}}=\frac{\vartheta \times D_{\mathrm{L}}}{(1+z)^{2}}$.

Here $D_{\mathrm{A}}$ is the angular diameter distance and $\vartheta=6 . ' 2$ is the angular size of our source J0808 as derived from the radio image shown in Fig. 1. This results in a linear size of $l=26.29 \mathrm{kpc}$, which can be compared to the size values found for other sources, as given in, e.g., Wang et al. (2011). The comparison is shown in Fig. 5, and we see that the J0808 is a relatively compact source compared to other galaxies at similar redshift.

In Fig. 6 we compare our target galaxy to the FRII galaxy 3C 438. We selected 3C438 for comparison since it is similar in apparent shape compared to J0808. In both sources we see in general an increasing intensity distribution from the center towards the edges. Hence the radio structure of SDSS J080800.99+483807.7 is indicative of an edge-brightened FRII source. 


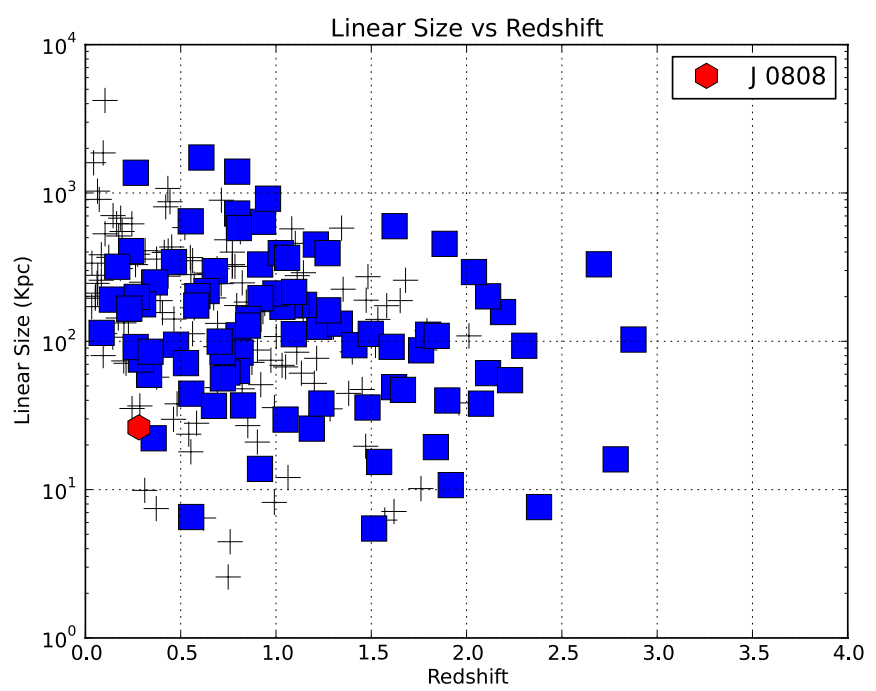

Fig. 5. Linear size versus redshift. Our target source is shown by the red hexagon, the plus signs represent the location of the 3CRR sources, the square signs represent the 7CRS sources (Wang et al. 2011).

\subsection{Radio loudness}

Our source is a prominent radio source. The differentiation between radio loud (RL) and radio quiet (RQ) sources primarily reflects the different importance of strong emission from radio jets and/or lobes (e.g., Yuan et al. 2008).

Kellermann et al. (1981) introduced a formalism to derive radio loudness by comparing the flux density at $5 \mathrm{GHz}$ to the flux density at $4400 \AA$ :

$R^{*}=\frac{S_{5 \mathrm{GHz}}}{S_{4400 \AA}}$.

Currently, there is no flux density measurement at $5 \mathrm{GHz}$, yet we do have several flux measurements at different frequencies, as shown in Table 3. From those, we estimate the spectral index via (cf. O’Dea et al. 2009)

$\alpha_{v 2}^{v 1}=\frac{\log \frac{S_{v 1}}{S_{v 2}}}{\log \frac{v 1}{v 2}}$.

Here $S_{v 1}$ and $S_{v 2}$ are flux densities at frequencies $v_{1}$ and $v_{2}$. From the data at $4.85 \mathrm{GHz}$ and $365 \mathrm{MHz}$ in Table 3, we find a spectral index of $\alpha=-0.80$. This indicates a dominant contribution to the radio flux from optically thin synchrotron radiation. Assuming a power law spectrum with $S_{v}=$ const. $\times v^{\alpha}$, we find a rest-frame $5 \mathrm{GHz}$ flux density of $S_{5 \mathrm{GHz}}=0.076 \mathrm{Jy}$. From our optical spectrum, we find a flux density at $4400 \AA$ of $S_{4400 \AA}=6.06 \times 10^{-6} \mathrm{Jy}$. This result is an estimate of the radio loudness of $R^{*}=12657$ or $\log R^{*}=4.1$. We can also compare it to other objects following Blundell (2003). To do so, we need to calculate the radio luminosities at $5 \mathrm{GHZ}$ and $178 \mathrm{MHz}$ via

$L_{v}=4 \pi \times D_{\mathrm{L}}^{2} \times S_{v}$

and find:

$$
\begin{array}{cc}
\log \left(L_{5 \mathrm{GHz}} / \mathrm{erg} \mathrm{s}^{-1} \mathrm{~Hz}^{-1}\right) & =32.27 \\
\log \left(L_{5 \mathrm{GHz}} / \mathrm{W} \mathrm{Hz}^{-1} \mathrm{sr}^{-1}\right) & =25.16 \\
\log \left(L_{178 \mathrm{MHz}} / \mathrm{erg} \mathrm{s}^{-1} \mathrm{~Hz}^{-1}\right) & =33.83 \\
\log \left(L_{178 \mathrm{MHz}} / \mathrm{W} \mathrm{Hz}^{-1} \mathrm{sr}^{-1}\right) & =26.33 .
\end{array}
$$
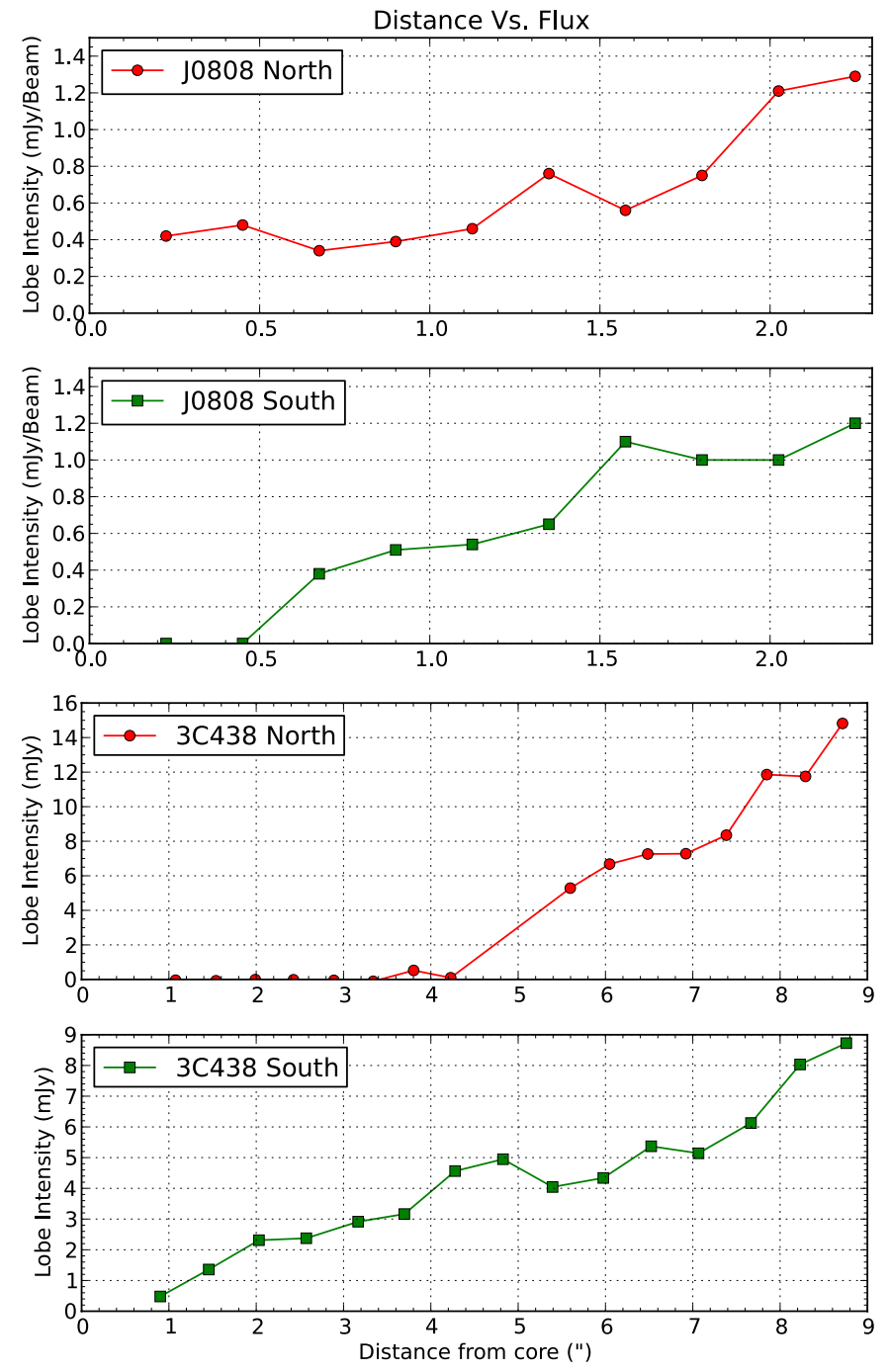

Fig. 6. Flux density values at different positions for each lobe (Northeast $\&$ Southwest) compared with other FRII sources (Treichel et al. 2001).

In Fig. 7, we show the comparison of our RL target with other sources. This places the J0808 among the RL objects. This general result does not change, even if we do the calculation only for the nuclear component, which is an order of magnitude weaker than the overall radio luminosity.

In Fig. 8 we show the radio spectrum of our target source. It can be represented as a straight power law. The fact that our flux density value at $18 \mathrm{~cm}$ wavelength lies below the power law fit is most likely due to resolution effects. It indicates that not all the flux density of the source was measured on the shortest baselines of our interferometric MERLIN observations.

\subsection{The central black hole of J0808}

There is a large body of evidence that indicates that most galaxies harbor a super-massive black hole at their centers (e.g., Magorrian et al. 1998; Ferrarese \& Merritt 2000; Gebhardt et al. 2000; Melia \& Falcke 2001; Ferrarese \& Ford 2005; Kormendy \& Ho 2013, and references therein). Here, we estimate the black hole mass of J0808 via the scaling relation between black hole mass and stellar velocity dispersion of the host bulge component. 
Table 3. Radio flux density at different frequencies.

\begin{tabular}{lcccc}
\hline \hline No. & $\begin{array}{c}\text { Observed passband } \\
\text { frequency } \\
(\mathrm{GHz})\end{array}$ & $\begin{array}{c}\text { Flux } \\
\text { density } \\
(\mathrm{Jy})\end{array}$ & $\begin{array}{c}\text { Luminosity } \\
\left(10^{25} \mathrm{~W} \mathrm{~Hz}^{-1}\right)\end{array}$ & References \\
\hline 1 & 4.85 & $0.077 \pm 0.010$ & 1.902 & 1 \\
2 & 4.85 & $0.075 \pm 0.011$ & 1.853 & 2 \\
3 & 1.66 & $0.088 \pm 0.004$ & 2.175 & This study \\
4 & 1.4 & $0.162 \pm 0.005$ & 4.003 & 3 \\
5 & 0.365 & $0.563 \pm 0.053$ & 13.913 & 4 \\
\hline
\end{tabular}

References. (1) Gregory \& Condon (1991); (2) Becker et al. (1991); (3) Condon et al. (1998); (4) Douglas et al. (1996).

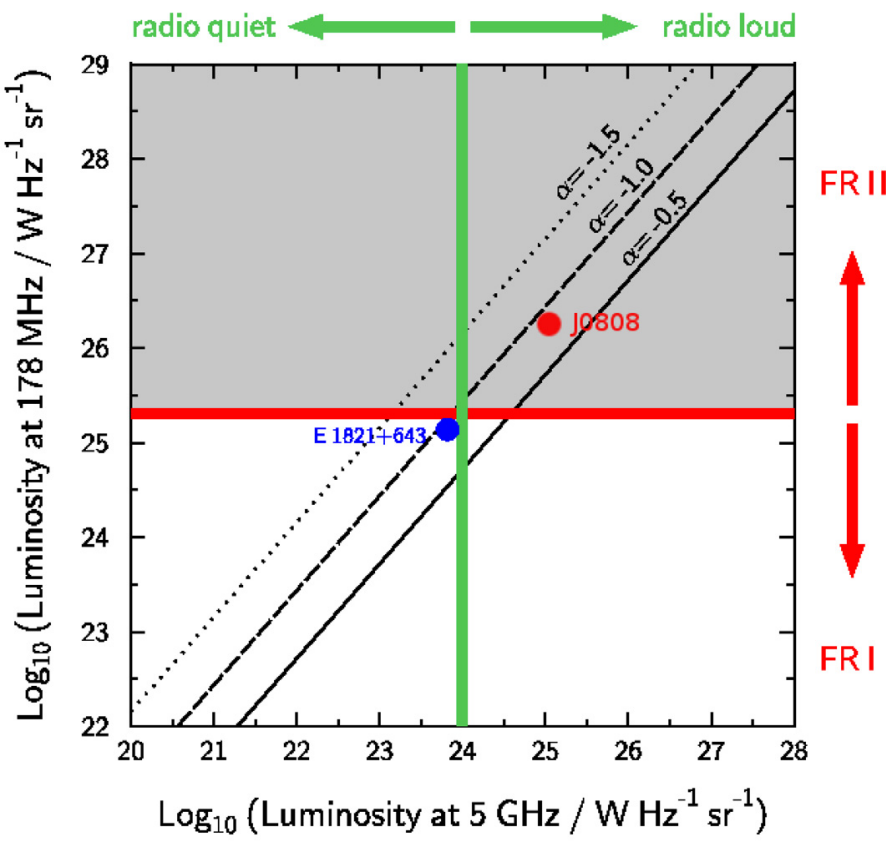

Fig. 7. Radio loudness scheme (Blundell 2003).

Following Ferrarese \& Merritt (2000), we used the relation

$\log \frac{M_{\mathrm{BH}}}{M_{\odot}}=8.12+\log \left(\frac{\sigma_{*}}{200 \mathrm{~km} \mathrm{~s}^{-1}}\right)^{4.24 \pm 0.41}$.

Assuming a Gaussian line shape and an instrumental resolution of $150 \mathrm{~km} \mathrm{~s}^{-1}$, we obtain a value for $\sigma_{*}$ via the following expression (Wang \& Zhang 2007; Greene \& Ho 2005):

$\sigma_{*}=\frac{\sqrt{\left(F W H M_{[\mathrm{O} \text { III }]}\right)^{2}-\left(150 \mathrm{~km} \mathrm{~s}^{-1}\right)^{2}} / 2.35}{1.34}$.

From Table 2, the FWHM of [O III] is $355 \mathrm{~km} \mathrm{~s}^{-1}$. Applying this value, we find $\sigma_{*}=102.2 \mathrm{~km} \mathrm{~s}^{-1}$ and a black hole mass of

$\log \left(\frac{M_{\mathrm{BH}}}{M_{\odot}}\right)=6.88 \pm 0.12$

The [O III] line strength turned out to exhibit large scatter in the scaling relation since in AGN the [O III] line emission is often associated with outflows from the active nucleus (Boroson 2003). The [S II] emission lines appear to be a better estimator of the underlying stellar velocity dispersion of the bulge (cf. Komossa \& Xu 2007). For comparison, we also determine

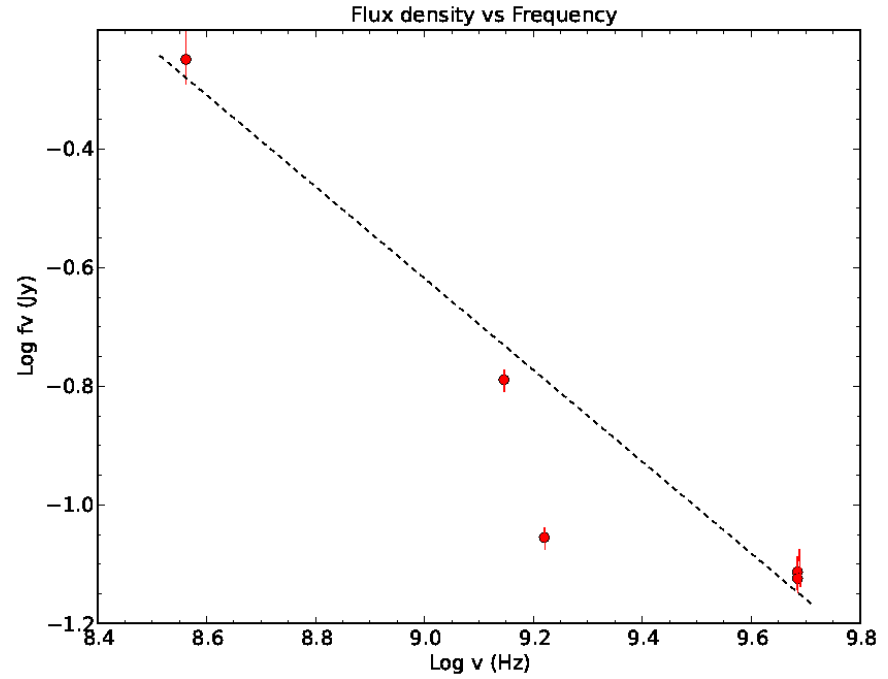

Fig. 8. Flux density and uncertainty versus frequency of J0808 from different surveys (Gregory \& Condon 1991; Becker et al. 1991; Condon et al. 1998; Douglas et al. 1996).

the black hole mass using the [S II] emission lines with the following equation:

$\sigma_{*}=\frac{\sqrt{\left(F W H M_{[\mathrm{S} \mathrm{II}]}\right)^{2}-\left(150 \mathrm{~km} \mathrm{~s}^{-1}\right)^{2}}}{2.35}$.

For the two [S II] lines in the spectrum (see Table 2), we obtain an average value of FWHM $([\mathrm{S} \mathrm{II}])_{\mathrm{avg}}=307 \mathrm{~km} \mathrm{~s}^{-1}$, resulting in a value of $\sigma_{*}=114.0 \mathrm{~km} \mathrm{~s}^{-1}$. Following Komossa \& Xu (2007) we find the estimation of black hole mass is

$\log \left(\frac{M_{\mathrm{BH}}}{M_{\odot}}\right)=6.9 \pm 0.15$.

Both methods result in a consistent estimate of the black hole mass of about $10^{7} M_{\odot}$.

The Eddington ratio $\eta$ is a measure of the accretion efficiency because it relates the observed AGN radiation output to that produced by maximum spherical accretion with isotropic radiation. The Eddington ratio $\eta=L_{\mathrm{bol}} / L_{\mathrm{edd}}$ is the ratio between the bolometric luminosity $L_{\text {bol }}$ and the Eddington luminosity $L_{\text {edd }}$. The bolometric luminosity is usually estimated from single-band measurements. Depending on the particular spectral energy distribution, there is considerable uncertainty in the bolometric correction for individual sources. Here, we estimate the bolometric luminosity, following the prescription from Vestergaard (2004), using the rest-frame $5100 \AA$ luminosity density

$L_{\mathrm{bol}} \approx 9.47 \times \lambda L_{\lambda}(5100 \AA) \mathrm{erg} \mathrm{s}^{-1}$. 


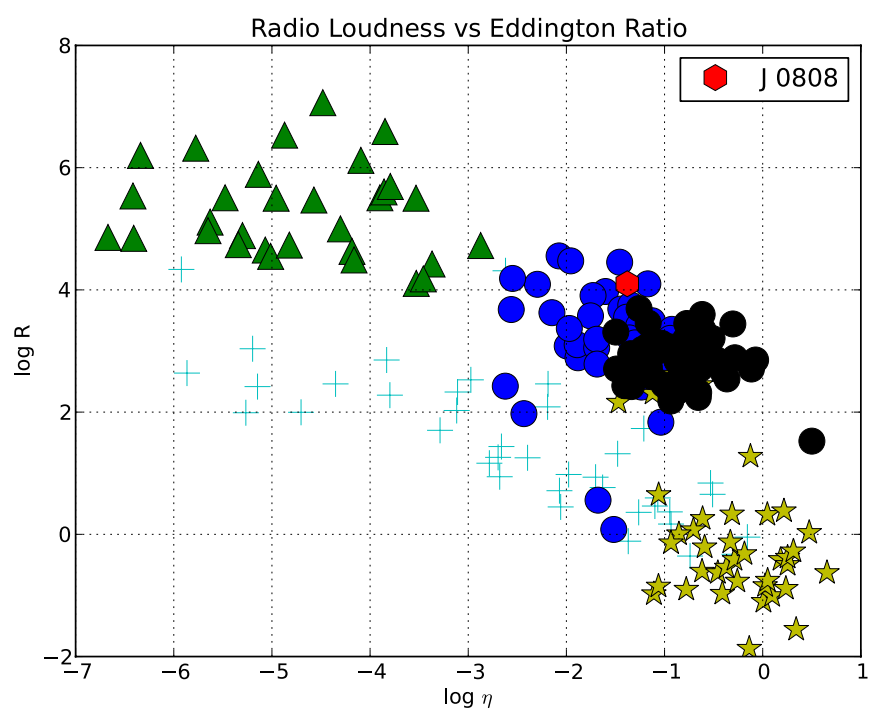

Fig. 9. Radio loudness (R) versus Eddington ratio $\eta$. Our target source is represented by a red hexagon, BLRGs are marked by the blue circles, radio loud quasars by the black circles, Seyfert galaxies and LINERs by the crosses, FR I radio galaxies by the green triangles, and PG quasars by the yellow stars (Sikora et al. 2007).

The rest-frame $5100 \AA$ luminosity density is estimated by fitting a linear combination of two simple starburst models, the AGN power law, and an extinction component to the observed spectrum (cf. Zuther et al. 2012). We find a $5100 \AA$ continuum luminosity of $\lambda L_{\lambda}(5100 \AA)=7.4 \times 10^{42} \mathrm{erg} \mathrm{s}^{-1}$. From Eq. (12), the bolometric luminosity is $L_{\mathrm{bol}} \approx 7.0 \times 10^{43} \mathrm{erg} \mathrm{s}^{-1}$, and with a black hole mass estimate of $\log \left(M_{\mathrm{BH}} / M_{\odot}\right) \sim 6.9$, the Eddington luminosity $^{2}$ is $L_{\mathrm{Edd}} \approx 1.6 \times 10^{45} \mathrm{erg} \mathrm{s}^{-1}$. This results in a value for the Eddington ratio of $\log \eta=-1.39$. In Figs. 9 and 10 we compare our values with published results. As shown in Fig. 9, in $\log \eta-\log R$ space, the source J0808 lies in the region shared by broad emission-line radio-galaxies (BLRG) and RL quasars. The same is found in Fig. $10\left(\log M_{\mathrm{BH}}-\log R\right)$. However, the mass of the super-massive black hole appears to be lower than that of the bulk of radio galaxies considered in the plot.

\subsection{Star formation}

The hosts of radio galaxies may be characterized by strong starformation activity. However, this is a matter of intense debate as interactions between the jet and the host ISM may also lead to suppression of star formation, as discussed in, e.g., Nesvadba et al. (2010). Radio galaxies with strong nuclear emission lines have star-formation activity that is a factor of 3-4 higher than in weak-line sources (Hardcastle et al. 2013). The line emission of our target source and the fact that it is RL suggests that it has a substantial star-formation rate.

To estimate the star-formation rate from optical data, we use the luminosity of the emission line [O II] $\lambda 3728$ (Hopkins et al. 2003; Kennicutt 1998; Ho 2005):

$S F R_{[\mathrm{O} \mathrm{II}]}\left[M_{\odot} \mathrm{yr}^{-1}\right]=\frac{L_{[\mathrm{OII}]}}{2.97 \times 10^{33} \mathrm{~W}}$.

Here we correct the reddening of the [O II] using theoretical Balmer decrement $\mathrm{H} \alpha / \mathrm{H} \beta=2.86$ and assuming case $\mathrm{B}$

\footnotetext{
2 The Eddington luminosity is the luminosity radiated at the Eddington limit and is calculated as $L_{\text {edd }} \approx 1.26 \times 10^{38}\left(\frac{M_{\mathrm{BH}}}{M_{\odot}}\right) \mathrm{erg} \mathrm{s}^{-1}$.
}

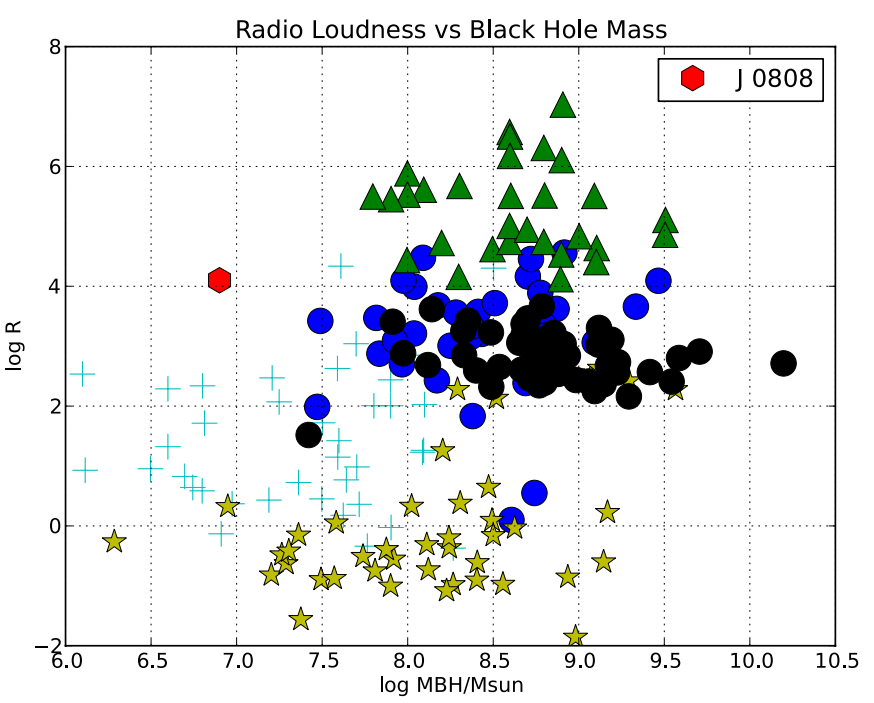

Fig. 10. Radio loudness versus black hole mass. See caption of Fig. 9.

recombination for a region with electron density of $10^{4} \mathrm{~cm}^{-3}$ and temperature $T=10^{4} \mathrm{~K}$ (Osterbrock 1989). According to the extinction law of Calzetti et al. (1994), we obtain the luminosity of [O II] $\lambda 3728$ of $5.82 \times 10^{34} \mathrm{~W}$, resulting in a starformation rate of $S F R=19.59 M_{\odot} \mathrm{yr}^{-1}$. In addition, we can make use of the WISE infrared survey data on J0808. There the source has been measured at 3.4, 4.6 and $12 \mu \mathrm{m}$. From a sample of dusty galaxies, we randomly picked galaxies from the IRAS point source catalog (Lonsdale \& Helou 1985) and find that the median flux density ratio between $12 \mu \mathrm{m}$ and $60 \mu \mathrm{m}$ wavelength lies at about 20 with an uncertainty of about 10 . From Helou (1986), we know that the ratio between the flux densities $S_{60}$ and $S_{100}$ at $100 \mu \mathrm{m}$ and $60 \mu \mathrm{m}$ wavelength is of the order of $2 \pm 1$. Hence, we can extrapolate from the WISE detection of J0808 at $12 \mu \mathrm{m}$ to obtain an estimate of the far-infrared (FIR) luminosity of $L_{\mathrm{FIR}}=23.96 \times 10^{5}\left(2.258 S_{60}+S_{100}\right) D_{\mathrm{L}}[\mathrm{Mpc}]^{2}=$ $(2.2 \pm 1.1) \times 10^{10} L_{\odot}$. This results in a star-formation rate of OBA stars of $v_{\mathrm{OBA}}=2.1 \times 10^{-10} \times L_{\mathrm{FIR}}=5 \pm 4 M_{\odot} \mathrm{yr}^{-1}$ (Thronson $\&$ Telesco 1986). To measure the star-formation rate from radio data, we apply the formalism published by Bell (2003), which is related to the $20 \mathrm{~cm}$ luminosity density

$\left(\frac{S F R}{M_{\odot} \mathrm{yr}^{-1}}\right)=5.52 \times 10^{-22}\left(\frac{L_{20 \mathrm{~cm}}}{\mathrm{~W} \mathrm{~Hz}^{-1}}\right)$.

From the radio spectrum discussed earlier, we obtain the luminosity at $20 \mathrm{~cm}$ and find $L_{20} \mathrm{~cm}=6 \times 10^{23} \mathrm{~W} \mathrm{~Hz}^{-1}$. Interpreting this radio luminosity in the context of star formation, this implies that only $1 \%$ of the total nuclear radio luminosity is required to explain the expected contribution from the above derived star-formation rate. The bulk of the emission may be due to the nuclear jet or the portion of the jet that flows back along the envelope of the jet onto the host.

The radio emission can also be due to contributions from supernova explosions. The rate of supernova explosions is a measure of the high-mass star formation. Following Condon \& Yin (1990), we calculate the supernova rate of our target galaxy from its central radio flux density via

$\left(\frac{S N R}{\mathrm{yr}^{-1}}\right) \approx 7.7 \times 10^{-24}\left(\frac{v}{\mathrm{GHz}}\right)^{\alpha}\left(\frac{L_{\mathrm{NT}}}{\mathrm{WHz}^{-1}}\right)$

Here, SNR is the supernova rate per year, $v$ is the frequency, $L_{\mathrm{NT}}$ the nonthermal radio luminosity, and $\alpha$ the radio spectral index. 
Y. E. Rashed et al.: SDSS J080800.99+483807.7

Table 4. Rest radio flux densities and luminosities for source components of the J0808 radio counterpart at $18 \mathrm{~cm}$.

\begin{tabular}{lcccccc}
\hline \hline Position & \multicolumn{2}{c}{ Brightest point } & \multicolumn{2}{c}{ Center } & \multicolumn{2}{c}{ Total area } \\
\hline & $\begin{array}{c}\text { Flux } \\
\left(10^{-3} \mathrm{Jy}\right. \\
\text { per beam })\end{array}$ & $\begin{array}{c}\text { Luminosity } \\
\left(10^{23} \mathrm{~W} \mathrm{~Hz}^{-1}\right. \\
\text { per beam })\end{array}$ & $\begin{array}{c}\text { Flux } \\
\left(10^{-3} \mathrm{Jy}\right. \\
\text { per beam })\end{array}$ & $\begin{array}{c}\text { Luminosity } \\
\left(10^{23} \mathrm{~W} \mathrm{~Hz}^{-1}\right. \\
\text { per beam })\end{array}$ & $\begin{array}{c}\text { Flux } \\
\left(10^{-2} \mathrm{Jy}\right)\end{array}$ & $\begin{array}{c}\text { Luminosity } \\
\left(10^{24} \mathrm{~W} \mathrm{~Hz}^{-1}\right)\end{array}$ \\
\hline Southeast Lobe & 1.28 & 3.17 & 0.81 & 2.01 & 3.71 & 9.17 \\
Center Region & 0.27 & 0.67 & - & - & 0.72 & 1.79 \\
Northwest Lobe & 1.41 & 3.49 & 0.45 & 1.12 & 4.69 & 11.59 \\
\hline
\end{tabular}

From our MERLIN observations at $18 \mathrm{~cm}$, we find a central luminosity at $18 \mathrm{~cm}$ of $L_{18 \mathrm{~cm}}=0.67 \times 10^{23} \mathrm{~W} \mathrm{~Hz}^{-1}$ from Table 4. For an assumed nuclear spectral index of $\alpha=-0.60$, we calculate the supernova rate at the center as $S N R \approx 0.4 \mathrm{yr}^{-1}$. This is consistent with the OBA star-formation rate obtained above from the extrapolation of the WISE $12 \mu \mathrm{m}$ measurements. However, given that the nuclear radio emission may contain a significant amount of nonthermal radiation from the jet and taking into account that the nuclear spectral index could also be steeper than $\alpha=-0.60$, we consider SNR as an upper limit. Following Hill et al. (2001) and Laine et al. (2006), the host is considered to be a Seyfert galaxy if the supernova rate is $\lesssim 1 \mathrm{yr}^{-1}$. Starburst galaxies have supernova rates of $S N R \lesssim 10 \mathrm{yr}^{-1}$.

\section{Discussion and conclusions}

Through MERLIN radio observations we have shown that the source J0808 is a compact double lobed radio source at a redshift of $z=0.2805 \pm 0.0003$; it shows a radio structure reminiscent of those that may present significant back-flow of material along the jet or outflow into the host (Wagner et al. 2013; Silk 2013). The host of the radio source has been detected in the optical and infrared emission in the SDSS and WISE surveys. The optical spectrum as well as the optical and infrared images of the host suggest that it is a compact early-type host galaxy. Our LBT MODS1R spectra, an estimate of the FIR luminosity, and the radio flux at the position of the host reveal that the host shows indications of strong star-forming activity. The widths of forbidden lines indicate a black hole mass of $\sim 10^{6.9} \mathrm{M}_{\odot}$.

The detection of the binarity of sources also clearly depends on the size of the telescope or interferometer used. In order to make sure that the location of J0808 at the lower edge of the distribution shown in Fig. 5 is indeed special, we compare the properties of small and large radio double sources that, in fact, form a continuum in separations. O'Dea \& Baum (1997) plot power versus projected largest linear size for the complete samples of gigahertz-peaked spectrum (GPS) and compact-steep spectrum (CSS) sources (Stanghellini et al. 1998; Fanti 2009; Fanti et al. 1995, 1985) and the 3CR (Laing et al. 1983) for the redshift range $0.2 \leq z \leq 1.0$ and find that there is a good overlap between the samples.

The radio source evolution can be constrained by plotting the number of sources as a function of size in the power versus linear size plane. O'Dea \& Baum (1997) find that the number of sources is roughly constant per linear size bin for sources less than about $10 \mathrm{kpc}$. For the larger sources, the number increases with increasing source size. This can be interpreted as an indication for a qualitatively different evolution of the small sources compared to the large ones. In fact, O'Dea (1998) points out that the GPS sources are entirely contained within the extent of the narrow-line region (less than about $1 \mathrm{kpc}$ ), while the CSS sources are contained entirely within the host galaxy (less than about $15 \mathrm{kpc}$ ). An explanation for this effect may be that the small sources are still embedded in the ISM of the host while the large ones can expand more freely. This shows that the source we are presenting here is indeed representative for the smaller (around $10 \mathrm{kpc}$ size) of the large double sources discussed by O’Dea \& Baum (1997).

Although the hosts of double radio sources in general do not have significant amounts of molecular gas, they do show signatures of star formation. An exception, on the one hand, are starburst radio galaxies that comprise 15-25 per cent of all powerful extragalactic radio sources (Tadhunter et al. 2011). On the other hand, it appears that the interaction with the radio jet quenches star formation and that hosts of radio galaxies seem to be inefficiently forming stars (e.g., Nesvadba et al. 2010). O'Dea et al. (2005) find no abundant molecular gas reservoirs in GPS radio sources with upper limits of $10^{9}$ to a few times $10^{10} M_{\odot}$.

Despite the apparent lack of large amounts of molecular gas, Fanti et al. (2011) and Labiano et al. (2008) find that most of the hosts of CSS radio binaries show an excess of ultraviolet (UV) radiation compared to the spectra of local RQ ellipticals. This UV excess may be due to an active nucleus or to a young stellar population, both of which may be triggered by the same event or influence each other. Similarly, we also find for J0808 diagnostic line ratios that clearly indicate the presence of star formation in the host galaxy. While a merger event may have caused both the ongoing jet activity, we have, however, no indication that the host has been affected by a recent merger event from the available imaging information. Hence the case of J0808 also allows us to follow a different interpretation: This source has an extended nuclear radio structure suggesting an interaction of the host ISM with the back-flowing material. Thus for J0808 the star formation may be triggered by the back-flow along the jet and its interaction with the AGN host.

Acknowledgements. The authors kindly thank the entire MERLIN staff for excellent support. We also thank the anonymous referee for the useful comments and suggestions that helped to improve the paper. Y.E. Rashed is supported by the German Academic Exchange Servis (DAAD) and by the Iraqi ministry of higher education and scientific research. G.B. is member of the Bonn-Cologne Graduate School of Physics and Astronomy (BCGS) and acknowledges support from the Konrad-Adenauer-Stiftung (KAS). M.V.-S. thanks the funding from the European Union Seventh Framework Programme (FP7/2007-2013) under grant agreement No. 312789. M.V. is member of the International Max-Planck Research School (IMPRS) for Astronomy and Astrophysics at the Universities of Bonn and Cologne. The work is also supported in part by the Deutsche Forschungsgemeinschaft (DFG) via grant SFB 956. We had fruitful discussions with members of the European Union funded COST Action MP0905: Black Holes in a violent Universe and the COST Action MP1104: Polarization as a tool to study the Solar System and beyond. This work has benefited from research funding from the European Community's sixth Framework Programme under RadioNet R113CT 2003 5058187. Radio observations were made with MERLIN, a National Facility operated by the University of Manchester at Jodrell Bank Observatory on behalf of STFC. The spectroscopic observations reported here were obtained at the LBT Observatory, a joint facility of the Smithsonian Institution and the University of Arizona. LBT observations were obtained as part of the Rat Deutscher Sternwarten guaranteed time on Steward Observatory facilities through the LBTB coorperation. This paper uses data taken with the MODS spectrographs built with funding from NSF grant 
AST-9987045 and the NSF Telescope System Instrumentation Program (TSIP), with additional funds from the Ohio Board of Regents and the Ohio State University Office of Research. The Sloan Digital Sky Survey is a joint project of the University of Chicago, Fermilab, the Institute for Advanced Study, the Japan Participation Group, Johns Hopkins University, the Max-Planck-Institute for Astronomy, the Max-Planck-Institute for Astrophysics, New Mexico State University, Princeton University, the United States Naval Observatory, and the University of Washington. Apache Point Observatory, site of the SDSS, is operated by the Astrophysical Research $\mathrm{C}$ onsortium. Funding for the project has been provided by the Alfred P. Sloan Foundation, the SDSS member institutions, NASA, the NSF, the Department of Energy, the Japanese Monbukagakusho, and the Max-Planck Society. The SDSS Web site is http: //www . sdss .org. Y.E.R. wants to thank all group members at I. Institute of Physics as well as Dr. Peter A. Schuller for fruitful discussions and help in the preparation of this publication.

\section{References}

Abazajian, K. N., Adelman-McCarthy, J. K., Agüeros, M. A., et al. 2009, ApJS, 182,543

Becker, R. H., White, R. L., \& Edwards, A. L. 1991, ApJS, 75, 1

Begelman, M. C., Blandford, R. D., \& Rees, M. J. 1984, Rev. Mod. Phys., 56, 255

Bell, E. F. 2003, ApJ, 586, 794

Blandford, R. D., \& Payne, D. G. 1982, MNRAS, 199, 883

Blandford, R. D., \& Znajek, R. L. 1977, MNRAS, 179, 433

Blundell, K. M. 2003, New Astron. Rev., 47, 593

Boroson, T. A. 2003, ApJ, 585, 647

Bridle, A. H., \& Perley, R. A. 1984, ARA\&A, 22, 319

Brocksopp, C., Kaiser, C. R., Schoenmakers, A. P., \& de Bruyn, A. G. 2011, MNRAS, 410, 484

Calzetti, D., Kinney, A. L., \& Storchi-Bergmann, T. 1994, ApJ, 429, 582

Condon, J. J., \& Yin, Q. F. 1990, ApJ, 357, 97

Condon, J. J., Cotton, W. D., Greisen, E. W., et al. 1998, AJ, 115, 1693

Douglas, J. N., Bash, F. N., Bozyan, F. A., Torrence, G. W., \& Wolfe, C. 1996, AJ, 111, 1945

Eales, S. A. 1985, MNRAS, 217, 179

Fabian, A. C. 2012, ARA\&A, 50, 455

Fanaroff, B. L., \& Riley, J. M. 1974, MNRAS, 167, 31P

Fanti, R. 2009, Astron. Nachr., 330, 303

Fanti, C., Fanti, R., Parma, P., Schilizzi, R. T., \& van Breugel, W. J. M. 1985, A\&A, 143, 292

Fanti, C., Fanti, R., Dallacasa, D., et al. 1995, A\&A, 302, 317

Fanti, C., Fanti, R., Zanichelli, A., Dallacasa, D., \& Stanghellini, C. 2011, A\&A, 528, A110

Ferrarese, L., \& Ford, H. 2005, Space Sci. Rev., 116, 523

Ferrarese, L., \& Merritt, D. 2000, ApJ, 539, L9

Gebhardt, K., Bender, R., Bower, G., et al. 2000, ApJ, 539, L13

Gopal-Krishna, Biermann, P. L., Gergely, L. Á., \& Wiita, P. J. 2012, Res. Astron. Astrophys., 12, 127

Greco, J. P., Martini, P., \& Thompson, T. A. 2012, ApJ, 757, 24

Greene, J. E., \& Ho, L. C. 2005, ApJ, 627, 721

Gregory, P. C., \& Condon, J. J. 1991, ApJS, 75, 1011

Hardcastle, M. J., Ching, J. H. Y., Virdee, J. S., et al. 2013, MNRAS, 429, 2407

Helou, G. 1986, ApJ, 311, L33

Hill, T. L., Heisler, C. A., Norris, R. P., Reynolds, J. E., \& Hunstead, R. W. 2001, AJ, 121, 128

Ho, L. C. 2005, ApJ, 629, 680

Hodges-Kluck, E. J., \& Reynolds, C. S. 2011, ApJ, 733, 58

Hogg, D. W. 1999, unpublished [arXiv: astro-ph/9905116]

Hopkins, A. M., Miller, C. J., Nichol, R. C., et al. 2003, ApJ, 599, 971

Juneau, S., Dickinson, M., Alexander, D. M., \& Salim, S. 2011, ApJ, 736, 104

Kauffmann, G., Heckman, T. M., Tremonti, C., et al. 2003, MNRAS, 346, 1055
Kellermann, K. I., Downes, A. J. B., Pauliny-Toth, I. I. K., et al. 1981, A\&A, 97, L1

Kennicutt, Jr., R. C. 1998, ARA\&A, 36, 189

Kewley, L. J., Dopita, M. A., Sutherland, R. S., Heisler, C. A., \& Trevena, J. 2001, ApJ, 556, 121

Kewley, L. J., Geller, M. J., \& Jansen, R. A. 2003, in BAAS 35, Am. Astron. Soc. Meet. Abstracts, \#119.01

Kewley, L. J., Groves, B., Kauffmann, G., \& Heckman, T. 2006, MNRAS, 372, 961

Komossa, S., \& Xu, D. 2007, ApJ, 667, L33

Kormendy, J., \& Ho, L. C. 2013, ARA\&A, in press [arXiv: 1304. 7762]

Krabbe, A., Sternberg, A., \& Genzel, R. 1994, ApJ, 425, 72

Kunert-Bajraszewska, M., \& Thomasson, P. 2009, Astron. Nachr., 330, 210

Labiano, A., O'Dea, C. P., Barthel, P. D., de Vries, W. H., \& Baum, S. A. 2008, A\&A, 477, 491

Laine, S., Kotilainen, J. K., Reunanen, J., Ryder, S. D., \& Beck, R. 2006, AJ, 131,701

Laing, R. A., Riley, J. M., \& Longair, M. S. 1983, MNRAS, 204, 151

Lamareille, F. 2010, A\&A, 509, A53

Lamareille, F., Mouhcine, M., Contini, T., Lewis, I., \& Maddox, S. 2004, MNRAS, 350, 396

Landt, H., Cheung, C. C., \& Healey, S. E. 2010, MNRAS, 408, 1103

Leahy, J. P., \& Williams, A. G. 1984, MNRAS, 210, 929

Lonsdale, C. J., \& Helou, G. 1985, Cataloged galaxies and quasars observed in the IRAS survey (Jet Propulsion Laboratory)

Magorrian, J., Tremaine, S., Richstone, D., et al. 1998, AJ, 115, 2285

Melia, F., \& Falcke, H. 2001, ARA\&A, 39, 309

Neeser, M. J., Eales, S. A., Law-Green, J. D., Leahy, J. P., \& Rawlings, S. 1995, ApJ, 451, 76

Nesvadba, N. P. H., Boulanger, F., Salomé, P., et al. 2010, A\&A, 521, A65

O'Dea, C. P. 1998, PASP, 110, 493

O'Dea, C. P., \& Baum, S. A. 1997, AJ, 113, 148

O'Dea, C. P., Gallimore, J., Stanghellini, C., Baum, S. A., \& Jackson, J. M. 2005, AJ, 129, 610

O’Dea, C. P., Daly, R. A., Kharb, P., Freeman, K. A., \& Baum, S. A. 2009, A\&A, 494,471

Oort, M. J. A., Katgert, P., \& Windhorst, R. A. 1987, Nature, 328, 500

Osterbrock, D. E. 1989, Astrophysics of gaseous nebulae and active galactic nuclei (University Science Books)

Osterbrock, D. E., Fulbright, J. P., Martel, A. R., et al. 1996, PASP, 108, 277

Osterbrock, D. E., Fulbright, J. P., \& Bida, T. A. 1997, PASP, 109, 614

Pogge, R. W., Atwood, B., Brewer, D. F., et al. 2010, in SPIE Conf. Ser., 7735

Pogge, R. W., Atwood, B., O'Brien, T. P., et al. 2012, in SPIE Conf. Ser., 8446

Sikora, M., Stawarz, Ł., \& Lasota, J.-P. 2007, ApJ, 658, 815

Silk, J. 2013, ApJ, 772, 112

Spergel, D. N., Verde, L., Peiris, H. V., et al. 2003, ApJS, 148, 175

Stanghellini, C., O’Dea, C. P., Dallacasa, D., et al. 1998, A\&AS, 131, 303

Tadhunter, C., Holt, J., González Delgado, R., et al. 2011, MNRAS, 412, 960

Thronson, Jr., H. A., \& Greenhouse, M. A. 1988, ApJ, 327, 671

Thronson, Jr., H. A., \& Telesco, C. M. 1986, ApJ, 311, 98

Treichel, K., Rudnick, L., Hardcastle, M. J., \& Leahy, J. P. 2001, ApJ, 561, 691

Veilleux, S., \& Osterbrock, D. E. 1987, ApJS, 63, 295

Vestergaard, M. 2004, ApJ, 601, 676

Vitale, M., Zuther, J., García-Marín, M., et al. 2012, A\&A, 546, A17

Vitale, M., Mignoli, M., Cimatti, A., et al. 2013, A\&A, 556, A11

Wagner, A. Y., \& Bicknell, G. V. 2011, ApJ, 728, 29

Wagner, A. Y., Umemura, M., \& Bicknell, G. V. 2013, ApJ, 763, L18

Wang, J.-M., \& Zhang, E.-P. 2007, ApJ, 660, 1072

Wang, Y., Knigge, C., Croston, J. H., \& Pavlovski, G. 2011, MNRAS, 418, 1138

Wright, E. L., Eisenhardt, P. R. M., Mainzer, A. K., et al. 2010, AJ, 140, 1868

York, D. G., Adelman, J., Anderson, Jr., J. E., et al. 2000, AJ, 120, 1579

Yuan, W., Zhou, H. Y., Komossa, S., et al. 2008, ApJ, 685, 801

Zuther, J., Fischer, S., \& Eckart, A. 2012, A\&A, 543, A57 